\title{
Efficient Synthesis of Pyrazinoic Acid Hybrid Conjugates
}

\author{
Siva S. Panda*a (D) \\ Abdullah M. Asirib \\ Mohamed Elagawanyc \\ Devan D. Buchanana \\ Behrad Torkian ${ }^{a}$ \\ Keerthana Bathala ${ }^{a}$ \\ Sean J. Thomas ${ }^{a}$ \\ Jason E. Capito ${ }^{a}$ \\ Muhammad N. Arshad ${ }^{b}$ \\ Abeer N. Al-Romaizan ${ }^{b}$ \\ a Department of Chemistry \& Physics, Augusta University, \\ Augusta, GA 30912, USA \\ ${ }^{b}$ Chemistry Department, Faculty of Science, King Abdulaziz \\ University, Jeddah, 21589, Saudi Arabia \\ c Department of Pharmaceutical Chemistry, faculty of pharma- \\ cy, Damanhour University, Damanhour, Egypt \\ sspanda12@gmail.com \\ sipanda@augusta.edu
}

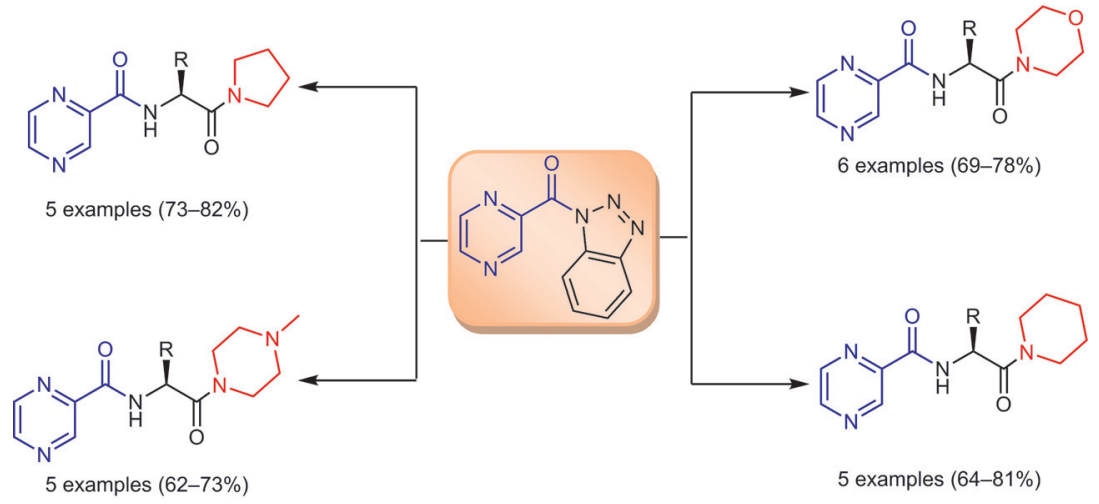

$\mathrm{R}=\mathrm{H}, \mathrm{CH}_{3}, \mathrm{CH}\left(\mathrm{CH}_{3}\right)_{2}, \mathrm{CH}\left(\mathrm{CH}_{3}\right) \mathrm{CH}_{2} \mathrm{CH}_{3}, \mathrm{CH}_{2} \mathrm{C}_{6} \mathrm{H}_{5}$
Received: 05.04.2017

Accepted after revision: 31.05.2017

Published online: 06.07.2017

DOI: 10.1055/s-0036-1590800; Art ID: so-2017-d0015-op

License terms: cc)

Abstract Benzotriazole-activated pyrazinoic acid was utilized as a versatile building block for the efficient and convenient synthesis of novel hybrid conjugates of pyrazinoic acid with secondary amines via amino acid linkers in high yields.

Key words pyrazinamide, pyrazinoic acid, secondary amines, amino acids, benzotriazole methodology, antituberculosis

Tuberculosis (TB) is a bacterial pathogen caused by Mycobacterium tuberculosis, which is known to cause pulmonary infection and to become extremely pervasive within the lungs. ${ }^{1-3} \mathrm{~TB}$ is considered to be one of the world's deadliest communicable diseases because of its high virulence and the ability of $M$. tuberculosis to enter into a dormant state, then subsequently undergo reactivation. ${ }^{3-5}$ Pyrazinamide (PZA) is a first-line antituberculosis prodrug that is often used in combinational therapy with drugs such as isoniazide, ethambutol, streptomycin, and rifampicin (Figure 1). ${ }^{6-8}$

PZA is perceived to inhibit vital ribosomal proteins after being converted into its active constituent, pyrazinoic acid (POA), by the tuberculosis enzyme, pyrazinamidase (PZAase) (Scheme 1). ${ }^{9}$ It may lower the $\mathrm{pH}$ of the area surrounding $M$. tuberculosis to such an extent that the organism is unable to grow. Due to its low lipophilicity, POA cannot be absorbed by the gastrointestinal tract. Fortunately, the drug can be absorbed in the pyrazinamide configuration.<smiles>NC(=O)c1cnccn1</smiles>

pyrazinamide<smiles>NNC(=O)c1ccncc1</smiles><smiles>CC[C@H](CO)NCCN[C@H](CC)CO</smiles>

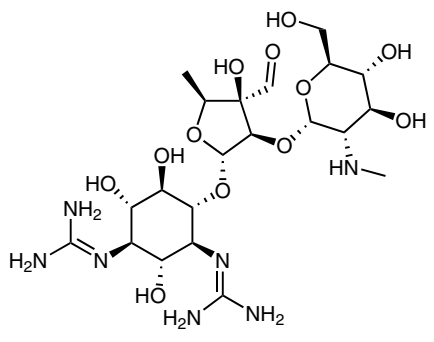

streptomycin

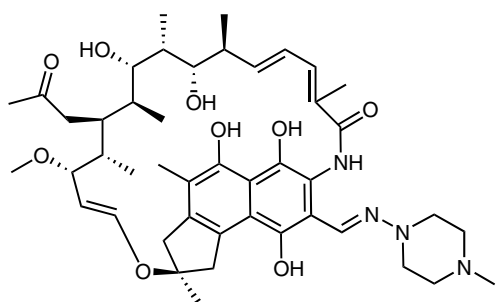

rifampicin

Figure 1 Current antituberculosis drugs 
<smiles>NC(=O)c1cnccn1</smiles>

PZA

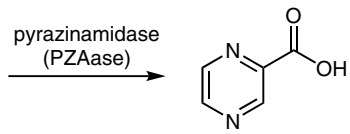

POA
Scheme 1 PZA is converted into POA in the presence of PZAase

One of the drawbacks of using PZA to treat TB is that it inhibits protein synthesis. With prolonged administration of the recommended dose, harmful side effects such as hepatitis, acute hypertension, thrombocytopenia, and gastrointestinal discomfort have been reported. ${ }^{10}$ To overcome these issues, several molecular hybridization approaches have been reported for the development of potential antitubercular agents. Most hybridized structures include clinically used drugs such as rifamycin, ethambutol and isoniazid coupled with other hydrophobic structures such as cinnamic acid derivatives. ${ }^{11-14}$ Unfortunately the most promising prodrugs of POA are not stable. ${ }^{15}$

Secondary amines such morpholine, piperidine, $\mathrm{N}$ methylpiperazine, and pyrrolidine are important scaffolds for potential biological molecules. Structural activity relationships (SARs) suggest that these secondary amines play an important role in bioactive molecules. ${ }^{16}$ Drug-amino acid conjugates are used to enhance drug delivery and to increase tissue cell penetration. In addition to acting as carriers of these agents, amino acids also amplify their bioavailability while maintaining their bioactive integrity. Recently, we have synthesized and reported several bioconjugates with enhanced biological properties and increased lipophilicity. ${ }^{17-20}$

In a continuation of our interest in synthesizing amino acid-peptide conjugates with biological significance, we report herein an efficient synthesis of pyrazimide hybrid conjugates, which contain various amino acids and secondary amines, that may decrease the dose of PZA required to fight $\mathrm{TB}$, increase the drug's lipophilicity and decrease adverse effects.

POA was activated as its benzotriazolide derivative 1 by following the previously reported procedure ${ }^{21}$ and coupled with free amino acids $\mathbf{2}$ in a mixture of acetonitrile and water $(7: 3)$ at $20{ }^{\circ} \mathrm{C}$ for $2 \mathrm{~h}$ in the presence of 1.5 equivalents of triethylamine to give compounds 3 . Attempts to prepare benzotriazole derivatives of POA-amino acid conjugates failed. We were also unsuccessful in coupling compounds 3 with secondary amines using different coupling reagents and ended up with mixtures of compounds as evidenced by TLC (Scheme 2). We therefore decided to redesign our approach and synthesize bis-conjugates of POA-secondary amines by coupling Boc-protected aminoacylbenzotriazoles $^{22}$ with secondary amines. After removing the Boc group with dioxane- $\mathrm{HCl}$, the unprotected conjugates were coupled with benzotriazole-activated POA to produce the desired products in good yields (Scheme 3). Boc-protected aminoacylbenzotriazoles $\mathbf{6 a - d}$ were treated with secondary amines 7-10 in the presence of triethylamine in acetonitrile at $20{ }^{\circ} \mathrm{C}$ for $2 \mathrm{~h}$ to obtain the conjugates in good yields without loss of chiral integrity (Scheme 3, Table 1).

Boc-protected amino acid-secondary amine conjugates 11a-t were deprotected using a dioxane- $\mathrm{HCl}$ mixture at $20{ }^{\circ} \mathrm{C}$ for $1 \mathrm{~h}$ to give unprotected amino acid-secondary amine conjugates 12a-t. These compounds were then used in the next step without further characterization. The target compounds 5a-t were prepared by coupling the unprotected amino acid-secondary amine conjugates with POAbenzotriazolide 1 in the presence of triethylamine in acetonitrile at $20^{\circ} \mathrm{C}$ for $3 \mathrm{~h}$ (Scheme 4 , Table 2). All compounds were fully characterized by NMR spectroscopy, HRMS and specific rotation. X-ray diffraction analysis of compound $\mathbf{5 a}$ further confirmed the formation of the hybrid conjugate (Figure 2).

In conclusion, benzotriazole-activated pyrazinoic acid has been used as a precursor for the efficient synthesis of pyrazinoic acid hybrid conjugates. The hybrid conjugates may be candidates for the development of new antituberculosis agents.

\section{Table 1 Boc-Protected Amino Acid-Secondary Amine Conjugates 11a-t}

\begin{tabular}{llllll}
\hline Entry & Product 11 & & Yield (\%) & Mp $\left({ }^{\circ} \mathrm{C}\right)$ & $\begin{array}{l}{[\alpha]_{\mathrm{D}}{ }^{20}(\mathrm{c} 1.0 \text { in }} \\
\text { MeOH })\end{array}$ \\
\hline 1 & Boc-Gly-Mor & $\mathbf{1 1 a}$ & 62 & $114-116$ & - \\
2 & Boc-L-Ala-Mor & $\mathbf{1 1 b}$ & 67 & oil & -20.3 \\
3 & Boc-DL-Ala-Mor & $\mathbf{1 1 b ^ { \prime }}$ & 74 & oil & racemic \\
4 & Boc-L-Val-Mor & $\mathbf{1 1 c}$ & 75 & $135-137$ & -17.5 \\
5 & Boc-L-lle-Mor & $\mathbf{1 1 d}$ & 81 & oil & -23.6 \\
6 & Boc-L-Phe-Mor & $\mathbf{1 1 e}$ & 84 & $129-131$ & -15.0 \\
7 & Boc-Gly-Pip & $\mathbf{1 1 f}$ & 90 & oil & - \\
8 & Boc-L-Ala-Pip & $\mathbf{1 1 g}$ & 97 & oil & -19.6 \\
9 & Boc-L-Val-Pip & $\mathbf{1 1 h}$ & 78 & oil & -20.6 \\
10 & Boc-L-lle-Pip & $\mathbf{1 1 i}$ & 79 & oil & -21.8 \\
11 & Boc-L-Phe-Pip & $\mathbf{1 1 j}$ & 81 & $122-124$ & -18.5 \\
12 & Boc-Gly-NMP & $\mathbf{1 1 k}$ & 76 & oil & - \\
13 & Boc-L-Ala-NMP & $\mathbf{1 1 l}$ & 81 & oil & -20.0 \\
14 & Boc-L-Val-NMP & $\mathbf{1 1 m}$ & 84 & oil & -14.3 \\
15 & Boc-L-lle-NMP & $\mathbf{1 1 n}$ & 69 & oil & -19.6 \\
16 & Boc-L-Phe-NMP & $\mathbf{1 1 0}$ & 83 & $111-113$ & -16.5 \\
17 & Boc-Gly-Pyr & $\mathbf{1 1 p}$ & 89 & oil & - \\
18 & Boc-L-Ala-Pyr & $\mathbf{1 1 q}$ & 83 & oil & -21.1 \\
19 & Boc-L-Val-Pyr & $\mathbf{1 1 r}$ & 67 & oil & -22.3 \\
20 & Boc-L-lle-Pyr & $\mathbf{1 1 s}$ & 72 & oil & -18.6 \\
21 & Boc-L-Phe-Pyr & $\mathbf{1 1 t}$ & 78 & $126-128$ & -24.0 \\
\hline & & & & & \\
\hline
\end{tabular}


<smiles>[R]C(NC(=O)c1cnccn1)C(=O)N[C@@H](CCC)C(=O)O</smiles>

2

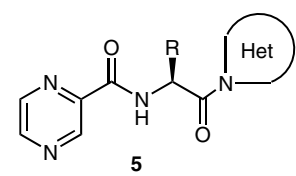

low yields and required further purification

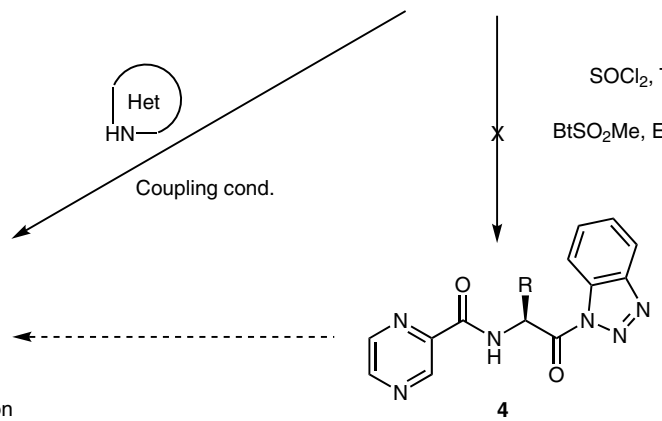
multiple spots on TLC

Scheme 2 Synthesis of POA hybrid conjugates with secondary heterocyclic amines and amino acid (Route I)

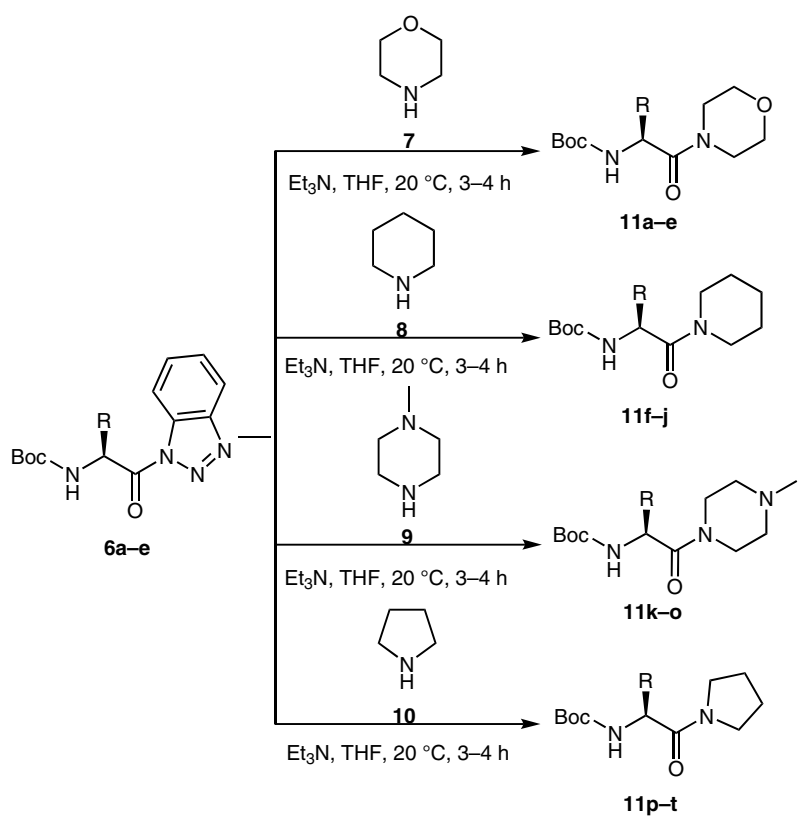

Scheme 3 Synthesis of Boc-protected amino acid-secondary heterocyclic amine conjugates

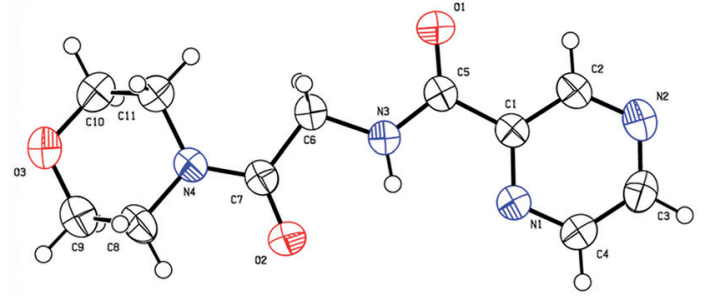

Figure 2 ORTEP diagram of POA-Gly-Mor (5a)

Melting points were determined with a capillary melting-point apparatus equipped with a digital thermometer. Reactions were monitored by using thin-layer chromatography (TLC) on $0.2 \mathrm{~mm}$ silica gel F254 plates (Merck). The chemical structures of final products and intermediates were characterized by ${ }^{1} \mathrm{H}$ and ${ }^{13} \mathrm{C}$ NMR spectroscopy with a Bruker NMR spectrometer (500 MHz, $125 \mathrm{MHz}$ ). ${ }^{13} \mathrm{C}$ NMR spectra were fully decoupled. Chemical shifts are reported in parts per million ( $\mathrm{ppm}$ ) using the deuterated solvent peak or tetramethylsilane as an internal standard. Mass spectrometric analysis was carried out with a high-resolution Biosystems QStar Elite time-of-flight electrospray mass spectrometer or an Agilent 6210 instrument using time-

$$
\text { Secondary Amine }=
$$

Scheme 4 Synthesis of POA hybrid conjugates with secondary heterocyclic amines and amino acid (Route II) 
Table 2 POA Hybrid Conjugates $\mathbf{5 a - t}$

\begin{tabular}{llllll}
\hline Entry & Product 5 & & Yield $(\%)$ & Mp $\left({ }^{\circ} \mathrm{C}\right)$ & $\begin{array}{l}{[\alpha]_{\mathrm{D}}{ }^{20}(\mathrm{c} 1.0 \text { in }} \\
\text { MeOH })\end{array}$ \\
\hline 1 & POA-Gly-Mor & $\mathbf{5 a}$ & 75 & $180-181$ & - \\
2 & POA-L-Ala-Mor & $\mathbf{5 b}$ & 69 & sticky & -25.4 \\
3 & POA-DL-Ala-Mor & $\mathbf{5 b}$ & 72 & oil & racemic \\
4 & POA-L-Val-Mor & $\mathbf{5 c}$ & 76 & oil & -19.2 \\
5 & POA-L-lle-Mor & $\mathbf{5 d}$ & 70 & oil & -20.3 \\
6 & POA-L-Phe-Mor & $\mathbf{5 e}$ & 78 & sticky & -25.6 \\
7 & POA-Gly-Pip & $\mathbf{5 f}$ & 74 & $123-125$ & - \\
8 & POA-L-Ala-Pip & $\mathbf{5 g}$ & 75 & oil & -19.9 \\
9 & POA-L-Val-Pip & $\mathbf{5 h}$ & 81 & oil & -23.6 \\
10 & POA-L-Ile-Pip & $\mathbf{5 i}$ & 64 & sticky & -22.0 \\
11 & POA-L-Phe-Pip & $\mathbf{5 j}$ & 79 & oil & -20.1 \\
12 & POA-Gly-NMP & $\mathbf{5 k}$ & 69 & sticky & - \\
13 & POA-L-Ala-NMP & $\mathbf{5 l}$ & 73 & oil & -26.5 \\
14 & POA-L-Val-NMP & $\mathbf{5 m}$ & 62 & oil & -20.9 \\
15 & POA-L-Ile-NMP & $\mathbf{5 n}$ & 64 & oil & -23.0 \\
16 & POA-L-Phe-NMP & $\mathbf{5 o}$ & 71 & oil & -28.1 \\
17 & POA-Gly-Pyr & $\mathbf{5 p}$ & 81 & $148-150$ & - \\
18 & POA-L-Ala-Pyr & $\mathbf{5 q}$ & 79 & oil & -23.0 \\
19 & POA-L-Val-Pyr & $\mathbf{5 r}$ & 82 & oil & -28.5 \\
20 & POA-L-Ile-Pyr & $\mathbf{5 s}$ & 74 & oil & -26.6 \\
21 & POA-L-Phe-Pyr & $\mathbf{5 t}$ & 73 & oil & -25.1 \\
\hline
\end{tabular}

of-flight mass spectrometry (TOF-MS) with electrospray ionization (ESI). Specific rotation measurements were carried out with an Autopol IV polarimeter.

\section{Synthesis of (1H-benzo[d][1,2,3]triazol-1-yl)(pyrazin-2-yl)metha- none (POA-Bt, 1$)^{21}$}

$1 H$-Benzotriazole (4.0 equiv) was dissolved in anhydrous methylene chloride. Thionyl chloride (1.2 equiv) was added and the mixture was stirred for $30 \mathrm{~min}$. Pyrazinoic acid (1.0 equiv) was added and the reaction mixture was stirred for $2-3 \mathrm{~h}$ at r.t. Upon completion of the reaction, $20 \%$ aqueous sodium bicarbonate was added and the organic layer was extracted twice with the alkaline solution, washed with brine, and dried over anhydrous magnesium sulfate. After filtration, the solvent was evaporated and the residue crystallized from diethyl ether to yield 1 in good yield.

\section{Synthesis of Secondary Amine-Amino Acid Conjugates 11a-t; Gen- eral Procedure}

A $50 \mathrm{~mL}$ round-bottom flask containing a small stir bar was charged with $N$-(Boc-aminoacyl)benzotriazole $\mathbf{6 a}-\mathbf{e}$ ( 1.0 equiv) and secondary amine (morpholine, piperidine, $N$-methylpiperazine, pyrrolidine) (1.0 equiv) dissolved in acetonitrile $(10 \mathrm{~mL})$ along with triethylamine (1.5 equiv). The reaction mixture was stirred at r.t. for $3-4 \mathrm{~h}$ and the progress of the reaction was monitored by TLC. After completion of the reaction, the acetonitrile was evaporated under reduced pressure and the residue was extracted with EtOAc. The organic layer was washed with aqueous sodium carbonate and dried over sodium sulfate. After filtration, the EtOAc was evaporated under reduced pressure to obtain the desired amino acid-secondary amine conjugate in good yield.

tert-Butyl (2-Morpholino-2-oxoethyl)carbamate (Boc-Gly-Mor, 11a)

Yield: $62 \%$; colorless microcrystals; $\mathrm{mp} 114-116{ }^{\circ} \mathrm{C}$.

${ }^{1} \mathrm{H} \mathrm{NMR}\left(500 \mathrm{MHz}, \mathrm{CDCl}_{3}\right): \delta=5.50($ br s, $1 \mathrm{H}), 3.95(\mathrm{~d}, J=3.5 \mathrm{~Hz}, 2 \mathrm{H})$, 3.70-3.62 (m, $6 \mathrm{H}), 3.40(\mathrm{t}, J=4.4 \mathrm{~Hz}, 2 \mathrm{H}), 1.45(\mathrm{~s}, 9 \mathrm{H})$.

${ }^{13} \mathrm{C}$ NMR $\left(125 \mathrm{MHz}, \mathrm{CDCl}_{3}\right): \delta=167.5,156.0,80.0,66.9,66.5,45.0$, 42.4, 42.3, 28.5.

HRMS (ESI): $m / z[M+H]^{+}$calcd for $\mathrm{C}_{11} \mathrm{H}_{20} \mathrm{~N}_{2} \mathrm{O}_{4}: 244.1424$; found: 244.1425 .

tert-Butyl (S)-(1-Morpholino-1-oxopropan-2-yl)carbamate (Boc-LAla-Mor, 11b)

Yield: 67\%; oil.

${ }^{1} \mathrm{H} \mathrm{NMR}\left(500 \mathrm{MHz}, \mathrm{CDCl}_{3}\right): \delta=5.51$ (br s, $1 \mathrm{H}$ ), 4.59 (br s, $1 \mathrm{H}$ ), 3.67$3.47(\mathrm{~m}, 8 \mathrm{H}), 1.43(\mathrm{~s}, 9 \mathrm{H}), 1.29(\mathrm{~d}, J=5.8 \mathrm{~Hz}, 3 \mathrm{H})$.

${ }^{13} \mathrm{C}$ NMR $\left(125 \mathrm{MHz}, \mathrm{CDCl}_{3}\right): \delta=171.5,155.3,79.8,67.0,66.8,46.1$, 42.6, 28.6, 19.5

HRMS (ESI): $m / z[M+H]^{+}$calcd for $\mathrm{C}_{12} \mathrm{H}_{22} \mathrm{~N}_{2} \mathrm{O}_{4}$ : 258.1584; found: 258.1580 .

tert-Butyl (1-Morpholino-1-oxopropan-2-yl)carbamate (Boc-DLAla-Mor, 11b')

Yield: 74\%; oil.

${ }^{1} \mathrm{H}$ NMR $\left(500 \mathrm{MHz}, \mathrm{CDCl}_{3}\right): \delta=5.57(\mathrm{~d}, J=5.8 \mathrm{~Hz}, 1 \mathrm{H}), 4.54(\mathrm{~d}$, $J=5.8 \mathrm{~Hz}, 1 \mathrm{H}), 3.62-3.40$ (m, $8 \mathrm{H}), 1.37$ (s, $9 \mathrm{H}), 1.24(\mathrm{~d}, J=7 \mathrm{~Hz}, 3 \mathrm{H})$.

${ }^{13} \mathrm{C}$ NMR $\left(125 \mathrm{MHz}, \mathrm{CDCl}_{3}\right): \delta=171.4,155.1,79.6,66.8,66.6,46.0$, 42.4, 28.4, 19.2 .

HRMS (ESI): $m / z[M+H]^{+}$calcd for $\mathrm{C}_{12} \mathrm{H}_{22} \mathrm{~N}_{2} \mathrm{O}_{4}$ : 258.1584; found: 258.1582 .

tert-Butyl (S)-(3-Methyl-1-morpholino-1-oxobutan-2-yl)carbamate (Boc-L-Val-Mor, 11c)

Yield: $75 \%$; colorless microcrystals; $\mathrm{mp} 135-137^{\circ} \mathrm{C}$.

${ }^{1} \mathrm{H}$ NMR $\left(500 \mathrm{MHz}, \mathrm{CDCl}_{3}\right): \delta=5.37(\mathrm{~d}, J=7.9 \mathrm{~Hz}, 1 \mathrm{H}), 4.34(\mathrm{t}, J=7.9 \mathrm{~Hz}$, $1 \mathrm{H}), 3.64-3.48(\mathrm{~m}, 8 \mathrm{H}), 1.89-1.83(\mathrm{~m}, 1 \mathrm{H}), 1.36(\mathrm{~s}, 9 \mathrm{H}), 0.88$ (d, $J=6.6 \mathrm{~Hz}, 3 \mathrm{H}), 0.83(\mathrm{~d}, J=6.6 \mathrm{~Hz}, 3 \mathrm{H})$.

${ }^{13} \mathrm{C}$ NMR $\left(125 \mathrm{MHz}, \mathrm{CDCl}_{3}\right): \delta=170.8,155.9,79.4,66.9,66.7,54.6$, 46.3, 42.4, 31.4, 28.3, 19.6, 17.3.

HRMS (ESI): $m / z[M+H]^{+}$calcd for $\mathrm{C}_{14} \mathrm{H}_{26} \mathrm{~N}_{2} \mathrm{O}_{4}$ : 286.1896; found: 286.1894 .

tert-Butyl ((2S,3S)-3-Methyl-1-morpholino-1-oxopentan-2-yl)carbamate (Boc-L-Ile-Mor, 11d)

Yield: $81 \%$; oil.

${ }^{1} \mathrm{H} \mathrm{NMR}\left(500 \mathrm{MHz}, \mathrm{CDCl}_{3}\right): \delta=5.32(\mathrm{~d}, J=7.9 \mathrm{~Hz}, 1 \mathrm{H}), 4.39(\mathrm{t}, J=7.9 \mathrm{~Hz}$, $1 \mathrm{H}), 3.68-3.48(\mathrm{~m}, 8 \mathrm{H}), 2.30-2.23(\mathrm{~m}, 1 \mathrm{H}), 1.65-1.64(\mathrm{~m}, 1 \mathrm{H}), 1.50-$ $1.45(\mathrm{~m}, 1 \mathrm{H}), 1.38(\mathrm{~s}, 9 \mathrm{H}), 0.88-0.81(\mathrm{~m}, 6 \mathrm{H})$.

${ }^{13} \mathrm{C}$ NMR $\left(125 \mathrm{MHz}, \mathrm{CDCl}_{3}\right): \delta=171.1,155.9,79.6,66.9,66.8,54.0$, $46.5,42.5,38.1,28.4,24.1,15.9,11.4$.

HRMS (ESI): $m / z[M+H]^{+}$calcd for $\mathrm{C}_{15} \mathrm{H}_{28} \mathrm{~N}_{2} \mathrm{O}_{4}$ : 300.2049; found: 300.2054 . 
tert-Butyl (S)-(1-Morpholino-1-oxo-3-phenylpropan-2-yl)carbamate (Boc-L-Phe-Mor, 11e)

Yield: $84 \%$; colorless microcrystals; $\mathrm{mp} 129-131{ }^{\circ} \mathrm{C}$.

${ }^{1} \mathrm{H} \mathrm{NMR}\left(500 \mathrm{MHz}, \mathrm{CDCl}_{3}\right): \delta=7.29-7.19(\mathrm{~m}, 5 \mathrm{H}), 5.44(\mathrm{~d}, J=7.5 \mathrm{~Hz}$, $1 \mathrm{H}), 4.82-4.78(\mathrm{~m}, 1 \mathrm{H}), 3.62-3.28(\mathrm{~m}, 6 \mathrm{H}), 3.05-2.89(\mathrm{~m}, 4 \mathrm{H}), 1.43$ (s, $9 \mathrm{H})$.

${ }^{13} \mathrm{C}$ NMR $\left(125 \mathrm{MHz}, \mathrm{CDCl}_{3}\right): \delta=170.5,155.2,136.5,129.7,128.8$, 127.3, 80.0, 66.7, 66.3, 51.0, 46.2, 42.4, 40.7, 28.5.

HRMS (ESI): $m / z[M+\mathrm{H}]^{+}$calcd for $\mathrm{C}_{18} \mathrm{H}_{26} \mathrm{~N}_{2} \mathrm{O}_{4}$ : 334.1898; found: 334.1909.

\section{tert-Butyl (2-0xo-2-(piperidin-1-yl)ethyl)carbamate (Boc-Gly-Pip,} 11f)

Yield: 90\%; oil.

${ }^{1} \mathrm{H} \mathrm{NMR}\left(500 \mathrm{MHz}, \mathrm{CDCl}_{3}\right.$ ): $\delta=5.56$ (br s, $1 \mathrm{H}$ ), 3.94 (d, J=3.5 Hz, $2 \mathrm{H}$ ), $3.56(\mathrm{t}, J=5 \mathrm{~Hz}, 2 \mathrm{H}), 3.31(\mathrm{t}, J=5 \mathrm{~Hz}, 2 \mathrm{H}), 1.65-1.63(\mathrm{~m}, 6 \mathrm{H}), 1.45$ (s, $9 \mathrm{H})$.

${ }^{13} \mathrm{C}$ NMR $\left(125 \mathrm{MHz}, \mathrm{CDCl}_{3}\right): \delta=167.9,164.3,79.6,45.9,44.2,41.9$, 28.4, 25.9, 24.8, 24.6.

HRMS (ESI): $m / z[M+H]^{+}$calcd for $\mathrm{C}_{12} \mathrm{H}_{22} \mathrm{~N}_{2} \mathrm{O}_{3}$ : 242.1633; found: 242.1641.

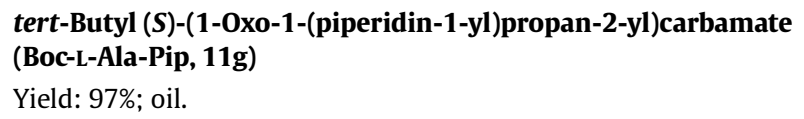

${ }^{13} \mathrm{C}$ NMR $\left(125 \mathrm{MHz}, \mathrm{CDCl}_{3}\right): \delta=171.0,155.3,79.5,46.6,46.4,43.4$, 28.6, 28.6, 28.5, 26.6, 25.7, 24.7, 19.7

HRMS (ESI): $m / z[\mathrm{M}+\mathrm{H}]^{+}$calcd for $\mathrm{C}_{13} \mathrm{H}_{24} \mathrm{~N}_{2} \mathrm{O}_{3}$ : 256.1787; found: 256.1792 .

\section{tert-Butyl (S)-(3-Methyl-1-oxo-1-(piperidin-1-yl)butan-2-yl)car- bamate (Boc-L-Val-Pip, 11h) \\ Yield: 78\%; oil.}

${ }^{1} \mathrm{H}$ NMR $\left(500 \mathrm{MHz}, \mathrm{CDCl}_{3}\right): \delta=5.37(\mathrm{~d}, J=7.5 \mathrm{~Hz}, 1 \mathrm{H}), 4.42(\mathrm{t}, J=7.5 \mathrm{~Hz}$, $1 \mathrm{H}), 3.54-3.41(\mathrm{~m}, 4 \mathrm{H}), 1.89-1.84(\mathrm{~m}, 1 \mathrm{H}), 1.60-1.45(\mathrm{~m}, 6 \mathrm{H}), 1.37$ (s, $9 \mathrm{H}), 0.90(\mathrm{~d}, J=6.7 \mathrm{~Hz}, 3 \mathrm{H}), 0.81(\mathrm{~d}, J=6.7 \mathrm{~Hz}, 3 \mathrm{H})$.

${ }^{13} \mathrm{C}$ NMR $\left(125 \mathrm{MHz}, \mathrm{CDCl}_{3}\right): \delta=170.8,155.9,79.4,66.9,66.7,54.6$, 46.3, 42.4, 31.4. 28.3, 19.6, 17.3.

HRMS (ESI): $m / z[\mathrm{M}+\mathrm{H}]^{+}$calcd for $\mathrm{C}_{15} \mathrm{H}_{28} \mathrm{~N}_{2} \mathrm{O}_{3}$ : 284.2105; found: 284.2100 .

tert-Butyl ((2S,3S)-3-Methyl-1-oxo-1-(piperidin-1-yl)pentan-2yl)carbamate (Boc-L-Ile-Pip, 11i)

Yield: 79\%; oil.

${ }^{1} \mathrm{H} \mathrm{NMR}\left(500 \mathrm{MHz}, \mathrm{CDCl}_{3}\right): \delta=5.33(\mathrm{~d}, J=7.9 \mathrm{~Hz}, 1 \mathrm{H}), 4.50(\mathrm{t}, J=7.9 \mathrm{~Hz}$, $1 \mathrm{H}), 3.59-3.49$ (m, $4 \mathrm{H}), 3.20-3.17$ ( $\mathrm{m}, 1 \mathrm{H}), 1.70-1.53(\mathrm{~m}, 8 \mathrm{H}), 1.43$ $(\mathrm{s}, 9 \mathrm{H}), 0.93(\mathrm{~d}, J=5.8 \mathrm{~Hz}, 3 \mathrm{H}), 0.88(\mathrm{t}, J=7.5 \mathrm{~Hz}, 3 \mathrm{H})$.

${ }^{13} \mathrm{C}$ NMR $\left(125 \mathrm{MHz}, \mathrm{CDCl}_{3}\right.$ ): $\delta=170.7,156.1,79.5,54.4,47.1,43.3$, $38.5,28.6,26.8,25.8,24.7,24.0,16.2,11.7$.

HRMS (ESI): $m / z[M+H]^{+}$calcd for $\mathrm{C}_{16} \mathrm{H}_{30} \mathrm{~N}_{2} \mathrm{O}_{3}$ : 298.2260; found: 298.2258 .
tert-Butyl (S)-(1-0xo-3-phenyl-1-(piperidin-1-yl)propan-2-yl)carbamate (Boc-L-Phe-Pip, 11j)

Yield: $81 \%$; colorless microcrystals; $\mathrm{mp} 122-124^{\circ} \mathrm{C}$.

${ }^{1} \mathrm{H}$ NMR $\left(500 \mathrm{MHz}, \mathrm{CDCl}_{3}\right): \delta=7.30-7.20(\mathrm{~m}, 5 \mathrm{H}), 5.48(\mathrm{~d}, J=8.5 \mathrm{~Hz}$, $1 \mathrm{H}), 4.89-4.85(\mathrm{~m}, 1 \mathrm{H}), 3.52-3.48(\mathrm{~m}, 2 \mathrm{H}), 3.28-3.23(\mathrm{~m}, 1 \mathrm{H}), 3.06-$ $2.97(\mathrm{~m}, 3 \mathrm{H}), 1.51-1.42(\mathrm{~m}, 15 \mathrm{H})$.

${ }^{13} \mathrm{C}$ NMR $\left(125 \mathrm{MHz}, \mathrm{CDCl}_{3}\right): \delta=169.9,155.2,136.8,129.8,128.6$, 127.0, 79.7, 51.1, 46.7, 43.2, 40.5, 28.5, 26.1, 25.5, 24.5 .

HRMS (ESI): $m / z[M+H]^{+}$calcd for $\mathrm{C}_{19} \mathrm{H}_{28} \mathrm{~N}_{2} \mathrm{O}_{3}$ : 332.2100; found: 332.2104.

\section{tert-Butyl (2-(4-Methylpiperazin-1-yl)-2-oxoethyl)carbamate} (Boc-Gly-NMP, 11k)

Yield: 76\%; oil.

${ }^{1} \mathrm{H}$ NMR (500 MHz, CDCl $)$ ): $\delta=5.54$ (br s, $1 \mathrm{H}$ ), 3.95 (d, J=4 Hz, $2 \mathrm{H}$ ), $3.64(\mathrm{t}, J=4.7 \mathrm{~Hz}, 2 \mathrm{H}), 3.40(\mathrm{t}, J=4.7 \mathrm{~Hz}, 2 \mathrm{H}), 2.41-2.38(\mathrm{~m}, 4 \mathrm{H}), 1.45$ (s, $9 \mathrm{H})$.

${ }^{13} \mathrm{C}$ NMR $\left(125 \mathrm{MHz}, \mathrm{CDCl}_{3}\right): \delta=169.7,164.4,79.4,54.7,54.5,45.9$, 44.2, 42.2, 41.9, 28.4.

HRMS (ESI): $m / z[M+H]^{+}$calcd for $\mathrm{C}_{12} \mathrm{H}_{23} \mathrm{~N}_{3} \mathrm{O}_{3}$ : 257.1739; found: 257.1740.

tert-Butyl (S)-(1-(4-Methylpiperazin-1-yl)-1-oxopropan-2-yl)carbamate (Boc-L-Ala-NMP, 111)

Yield: 81\%; oil.

${ }^{1} \mathrm{H}$ NMR $\left(500 \mathrm{MHz}, \mathrm{CDCl}_{3}\right): \delta=5.53(\mathrm{~d}, J=7.6 \mathrm{~Hz}, 1 \mathrm{H}), 4.55-4.50(\mathrm{~m}$, $1 \mathrm{H}), 3.63-3.40$ (m, $4 \mathrm{H}), 2.35-2.30(\mathrm{~m}, 4 \mathrm{H}), 2.23$ (s, $3 \mathrm{H}), 1.36(\mathrm{~s}, 9 \mathrm{H})$, $1.22(\mathrm{~d}, J=6.9 \mathrm{~Hz}, 1 \mathrm{H})$.

${ }^{13} \mathrm{C}$ NMR $\left(125 \mathrm{MHz}, \mathrm{CDCl}_{3}\right): \delta=171.2,155.2,79.6,55.1,54.7,46.2$, 46.0, 45.4, 42.0, 28.5, 19.5 .

HRMS (ESI): $m / z[M+H]^{+}$calcd for $\mathrm{C}_{13} \mathrm{H}_{25} \mathrm{~N}_{3} \mathrm{O}_{3}: 271.1896$; found: 271.1899.

tert-Butyl (S)-(3-Methyl-1-(4-methylpiperazin-1-yl)-1-oxobutan2-yl)carbamate (Boc-L-Val-NMP, 11m)

Yield: $84 \%$; oil.

${ }^{1} \mathrm{H}$ NMR $\left(500 \mathrm{MHz}, \mathrm{CDCl}_{3}\right): \delta=5.34(\mathrm{~d}, J=7.2 \mathrm{~Hz}, 1 \mathrm{H}), 4.30(\mathrm{~d}$, $J=7.2 \mathrm{~Hz}, 1 \mathrm{H}), 3.53-3.39$ (m, $4 \mathrm{H}), 2.27-2.22$ (m, $4 \mathrm{H}), 2.15(\mathrm{~s}, 3 \mathrm{H})$, $1.82-1.76(\mathrm{~m}, 1 \mathrm{H}), 1.29(\mathrm{~s}, 9 \mathrm{H}), 0.81(\mathrm{~d}, J=6.7 \mathrm{~Hz}, 3 \mathrm{H}), 0.74$ (d, $J=6.7 \mathrm{~Hz}, 3 \mathrm{H})$.

${ }^{13} \mathrm{C}$ NMR $\left(125 \mathrm{MHz}, \mathrm{CDCl}_{3}\right): \delta=170.4,155.8,79.3,55.2,54.7,45.9$, 45.6, 41.9, 31.5, 28.3, 19.7, 17.1 .

HRMS (ESI): $m / z[M+H]^{+}$calcd for $\mathrm{C}_{15} \mathrm{H}_{29} \mathrm{~N}_{3} \mathrm{O}_{3}$ : 299.2211; found: 299.2208 .

tert-Butyl ((2S,3S)-3-Methyl-1-(4-methylpiperazin-1-yl)-1-oxopentan-2-yl)carbamate (Boc-L-Ile-NMP, 11n)

Yield: 69\%; oil.

${ }^{1} \mathrm{H}$ NMR $\left(500 \mathrm{MHz}, \mathrm{CDCl}_{3}\right): \delta=5.26(\mathrm{~d}, J=8.2 \mathrm{~Hz}, 1 \mathrm{H}), 4.41(\mathrm{t}, J=8.2 \mathrm{~Hz}$, $1 \mathrm{H}), 3.66-3.46(\mathrm{~m}, 4 \mathrm{H}), 2.35-2.32(\mathrm{~m}, 4 \mathrm{H}), 2.24$ (s, $3 \mathrm{H}), 2.15-2.10$ $(\mathrm{m}, 1 \mathrm{H}), 1.65-1.60(\mathrm{~m}, 1 \mathrm{H}), 1.49-1.45(\mathrm{~m}, 1 \mathrm{H}), 1.29(\mathrm{~s}, 9 \mathrm{H}), 0.91-$ $0.80(\mathrm{~m}, 6 \mathrm{H})$.

${ }^{13} \mathrm{C}$ NMR (125 MHz, $\left.\mathrm{CDCl}_{3}\right): \delta=170.9,156.0,79.6,55.4,54.9,54.3$, 46.1, 45.9, 42.1, 38.4, 28.5, 24.1, 16.1, 11.6.

HRMS (ESI): $m / z[M+H]^{+}$calcd for $\mathrm{C}_{16} \mathrm{H}_{31} \mathrm{~N}_{3} \mathrm{O}_{3}: 313.2365$; found: 313.2366 . 
tert-Butyl (S)-(1-(4-Methylpiperazin-1-yl)-1-oxo-3-phenylpropan2-yl)carbamate (Boc-L-Phe-NMP, 110)

Yield: $83 \%$; white microcrystals; $\mathrm{mp} 111-113^{\circ} \mathrm{C}$.

${ }^{1} \mathrm{H} \mathrm{NMR}\left(500 \mathrm{MHz}, \mathrm{CDCl}_{3}\right): \delta=7.29-7.18(\mathrm{~m}, 5 \mathrm{H}), 5.45(\mathrm{~d}, J=8.2 \mathrm{~Hz}$, $1 \mathrm{H}), 4.85-4.80(\mathrm{~m}, 1 \mathrm{H}), 3.60-3.52(\mathrm{~m}, 2 \mathrm{H}), 3.34-3.28(\mathrm{~m}, 1 \mathrm{H}), 2.99-$ $2.92(\mathrm{~m}, 3 \mathrm{H}), 2.33-2.17(\mathrm{~m}, 7 \mathrm{H}), 1.42(\mathrm{~m}, 9 \mathrm{H})$.

${ }^{13} \mathrm{C}$ NMR $\left(125 \mathrm{MHz}, \mathrm{CDCl}_{3}\right): \delta=170.2,155.2,136.6,129.8,128.7$, 127.2, 79.9, 54.7, 54.5, 51.1, 46.0, 45.6, 42.0, 40.7, 28.6.

HRMS (ESI): $m / z[M+\mathrm{H}]^{+}$calcd for $\mathrm{C}_{19} \mathrm{H}_{29} \mathrm{~N}_{3} \mathrm{O}_{3}$ : 347.2211; found: 347.2220.

\section{tert-Butyl (2-0xo-2-(pyrrolidin-1-yl)ethyl)carbamate (Boc-Gly- Pyr, 11p)}

Yield: 89\%; oil.

${ }^{1} \mathrm{H} \mathrm{NMR}\left(500 \mathrm{MHz}, \mathrm{CDCl}_{3}\right): \delta=5.38$ (br s, $\left.1 \mathrm{H}\right), 4.07$ (d, J=4.3 Hz, $2 \mathrm{H}$ ), 3.56-3.38 (m, $4 \mathrm{H}), 2.03-1.84(\mathrm{~m}, 4 \mathrm{H})$.

${ }^{13} \mathrm{C}$ NMR $\left(125 \mathrm{MHz}, \mathrm{CDCl}_{3}\right): \delta=168.3,163.1,79.6,46.9,46.3,43.1$, $28.5,26.3,24.5$.

HRMS (ESI): $m / z[\mathrm{M}+\mathrm{H}]^{+}$calcd for $\mathrm{C}_{11} \mathrm{H}_{20} \mathrm{~N}_{2} \mathrm{O}_{3}$ : 228.1475; found: 228.1477.

\section{tert-Butyl (S)-(1-0xo-1-(pyrrolidin-1-yl)propan-2-yl)carbamate} (Boc-L-Ala-Pyr, 11q)

Yield: 83\%; oil.

${ }^{1} \mathrm{H}$ NMR (500 MHz, $\mathrm{CDCl}_{3}$ ): $\delta=5.48(\mathrm{br} \mathrm{s}, 1 \mathrm{H}), 4.47-4.42(\mathrm{~m}, 1 \mathrm{H})$, 3.63-3.40 (m, 4 H), 1.99-1.86 (m, 4 H), 1.43 (s, 9 H), 1.31 (d, J=6.7 Hz, $1 \mathrm{H})$.

${ }^{13} \mathrm{C}$ NMR $\left(125 \mathrm{MHz}, \mathrm{CDCl}_{3}\right): \delta=171.4,155.3,79.6,48.0,46.5,46.1$, 28.6, 26.2, 24.3, 18.9 .

HRMS (ESI): $m / z[M+H]^{+}$calcd for $\mathrm{C}_{12} \mathrm{H}_{22} \mathrm{~N}_{2} \mathrm{O}_{3}: 242.1630$; found: 242.1637.

\section{tert-Butyl (S)-(3-Methyl-1-oxo-1-(pyrrolidin-1-yl)butan-2-yl)car-} bamate (Boc-L-Val-Pyr, 11r)

Yield: 67\%; oil.

${ }^{1} \mathrm{H}$ NMR $\left(500 \mathrm{MHz}, \mathrm{CDCl}_{3}\right): \delta=5.26(\mathrm{~d}, \mathrm{~J}=7.8 \mathrm{~Hz}, 1 \mathrm{H}), 4.15(\mathrm{t}, J=7.8 \mathrm{~Hz}$, $1 \mathrm{H}), 3.60-3.30(\mathrm{~m}, 4 \mathrm{H}), 1.87-1.75(\mathrm{~m}, 5 \mathrm{H}), 1.37(\mathrm{~s}, 9 \mathrm{H}), 0.86(\mathrm{~d}$, $J=6.7 \mathrm{~Hz}, 3 \mathrm{H}), 0.83(\mathrm{~d}, J=6.7 \mathrm{~Hz}, 3 \mathrm{H})$.

${ }^{13} \mathrm{C}$ NMR $\left(125 \mathrm{MHz}, \mathrm{CDCl}_{3}\right): \delta=170.7,155.8,79.2,57.0,46.6,45.7$, 31.3, 28.3, 26.0, 24.1, 19.5, 17.5 .

HRMS (ESI): $m / z[M+H]^{+}$calcd for $\mathrm{C}_{14} \mathrm{H}_{26} \mathrm{~N}_{2} \mathrm{O}_{3}$ : 270.1945; found: 270.1946 .

tert-Butyl ((2S,3S)-3-Methyl-1-oxo-1-(pyrrolidin-1-yl)pentan-2yl)carbamate (Boc-L-Ile-Pyr, 11s)

Yield: 72\%; oil.

${ }^{1} \mathrm{H}$ NMR $\left(500 \mathrm{MHz}, \mathrm{CDCl}_{3}\right): \delta=5.23(\mathrm{~d}, J=8.5 \mathrm{~Hz}, 1 \mathrm{H}), 4.27(\mathrm{t}, J=8.5 \mathrm{~Hz}$, $1 \mathrm{H}), 3.74-3.69$ (m, $1 \mathrm{H}), 3.56-3.20$ ( $\mathrm{m}, 4 \mathrm{H}), 1.67-1.86$ (m, $4 \mathrm{H}), 1.73-$ 1.55 (m, $2 \mathrm{H}), 1.43$ (s, $9 \mathrm{H}), 0.93-0.86(\mathrm{~m}, 6 \mathrm{H})$.

${ }^{13} \mathrm{C}$ NMR $\left(125 \mathrm{MHz}, \mathrm{CDCl}_{3}\right): \delta=171.2,156.0,79.6,56.6,48.1,46.9$, 46.0, 38.2, 28.6, 26.2, 24.4, 15.8, 11.5.

HRMS (ESI): $m / z[M+H]^{+}$calcd for $\mathrm{C}_{15} \mathrm{H}_{28} \mathrm{~N}_{2} \mathrm{O}_{3}$ : 284.2101; found: 284.2111 .
tert-Butyl (S)-(1-0xo-3-phenyl-1-(pyrrolidin-1-yl)propan-2yl)carbamate (Boc-L-Phe-Pyr, 11t)

Yield: 78\%; colorless microcrystals; $\mathrm{mp} 126-128{ }^{\circ} \mathrm{C}$.

${ }^{1} \mathrm{H}$ NMR $\left(500 \mathrm{MHz}, \mathrm{CDCl}_{3}\right): \delta=7.29-7.22(\mathrm{~m}, 5 \mathrm{H}), 5.43(\mathrm{~d}, J=7.8 \mathrm{~Hz}$, $1 \mathrm{H}), 4.63-4.58(\mathrm{~m}, 1 \mathrm{H}), 3.48-3.33(\mathrm{~m}, 3 \mathrm{H}), 3.28-3.23(\mathrm{~m}, 1 \mathrm{H}), 3.01-$ 2.94 (m, 2 H), 2.63-2.57 (m, $1 \mathrm{H}), 1.82-1.56$ (m, $4 \mathrm{H}), 1.44$ (m, $9 \mathrm{H})$.

${ }^{13} \mathrm{C}$ NMR $\left(125 \mathrm{MHz}, \mathrm{CDCl}_{3}\right): \delta=170.1,155.3,136.8,129.7,128.5$, $127.0,79.8,53.8,46.4,45.9,40.5,28.6,26.0,24.2$.

HRMS (ESI): $m / z[\mathrm{M}+\mathrm{H}]^{+}$calcd for $\mathrm{C}_{18} \mathrm{H}_{26} \mathrm{~N}_{2} \mathrm{O}_{3}: 318.1944$; found: 318.1943.

\section{Synthesis of Bisconjugates 5a-t; General Procedure}

The secondary amine-amino acid conjugate was stirred in $4 \mathrm{M} \mathrm{HCl}-$ dioxane solution for $1 \mathrm{~h}$. The dioxane was evaporated under reduced pressure and the residue was treated with diethyl ether. The resulting solid was treated without further purification with the benzotriazole derivative of pyrazinoic acid in the presence of triethylamine (1.5 equiv) in acetonitrile $(10 \mathrm{~mL})$. The reaction mixture was stirred at 20 ${ }^{\circ} \mathrm{C}$ for 4-6 h, monitoring by TLC. Upon completion of reaction, the acetonitrile was evaporated and the residue was extracted with EtOAc. The organic layer was washed with aqueous sodium carbonate and dried over anhydrous sodium sulfate. After filtration, the EtOAc was evaporated under reduced pressure to obtain the desired conjugates in good yields.

\section{$\mathrm{N}$-(2-Morpholino-2-oxoethyl)pyrazine-2-carboxamide (POA-Gly-} Mor, 5a)

Yield: 75\%; colorless microcrystals; $\mathrm{mp} 180-181^{\circ} \mathrm{C}$.

${ }^{1} \mathrm{H} \mathrm{NMR}\left(500 \mathrm{MHz}, \mathrm{CDCl}_{3}\right.$ ): $\delta=9.32$ (s, $\left.1 \mathrm{H}\right), 8.70$ (br s, $1 \mathrm{H}$ ), 8.65 (br s, $1 \mathrm{H}), 8.53$ (br s, $1 \mathrm{H}), 4.23$ (d, J=4.3 Hz, $2 \mathrm{H}), 3.68-3.63$ (m, $6 \mathrm{H}), 3.46-$ $3.44(\mathrm{~m}, 2 \mathrm{H})$.

${ }^{13} \mathrm{C}$ NMR $\left(125 \mathrm{MHz}, \mathrm{CDCl}_{3}\right)$ : $\delta=166.4,163.3,147.5,144.3,143.1,66.8$, 66.5, 45.1, 42.5. 41.2.

HRMS (ESI): $m / z[M+H]^{+}$calcd for $\mathrm{C}_{11} \mathrm{H}_{14} \mathrm{~N}_{4} \mathrm{O}_{3}$ : 250.1068; found: 250.1074 .

(S)-N-(1-Morpholino-1-oxopropan-2-yl)pyrazine-2-carboxamide (POA-L-Ala-Mor, 5b)

Yield: 68\%; oil.

${ }^{1} \mathrm{H}$ NMR (500 MHz, $\mathrm{CDCl}_{3}$ ): $\delta=9.34(\mathrm{~s}, 1 \mathrm{H}), 8.74-8.70(\mathrm{~m}, 2 \mathrm{H}), 8.50$ (br s, $1 \mathrm{H}), 5.13-5.08(\mathrm{~m}, 1 \mathrm{H}), 3.69-3.53(\mathrm{~m}, 8 \mathrm{H}), 1.45(\mathrm{~d}, J=6.9 \mathrm{~Hz}$, $3 \mathrm{H})$.

${ }^{13} \mathrm{C}$ NMR $\left(125 \mathrm{MHz}, \mathrm{CDCl}_{3}\right): \delta=170.8,162.6,147.5,144.3,143.0,66.9$, 66.7, 46.2, 45.4, 42.8, 19.0.

HRMS (ESI): $m / z[\mathrm{M}+\mathrm{H}]^{+}$calcd for $\mathrm{C}_{12} \mathrm{H}_{16} \mathrm{~N}_{4} \mathrm{O}_{3}$ : 264.1221; found: 264.1225 .

(S)-N-(1-Morpholino-1-oxopropan-2-yl)pyrazine-2-carboxamide (POA-DL-Ala-Mor, 5b')

Yield: 72\%; gum.

${ }^{1} \mathrm{H}$ NMR $\left(500 \mathrm{MHz}, \mathrm{CDCl}_{3}\right): \delta=9.33(\mathrm{~s}, 1 \mathrm{H}), 8.74-8.68(\mathrm{~m}, 2 \mathrm{H}), 8.50$ (br s, $1 \mathrm{H}), 5.16-5.07(\mathrm{~m}, 1 \mathrm{H}), 3.69-3.55(\mathrm{~m}, 8 \mathrm{H}), 1.44(\mathrm{~d}, J=6.9 \mathrm{~Hz}$, $3 \mathrm{H})$.

${ }^{13} \mathrm{C}$ NMR $\left(125 \mathrm{MHz}, \mathrm{CDCl}_{3}\right): \delta=170.8,162.4,147.5,145.0,143.1,66.9$, 66.7, 46.2, 45.5, 42.7, 19.0.

HRMS (ESI): $m / z[\mathrm{M}+\mathrm{H}]^{+}$calcd for $\mathrm{C}_{12} \mathrm{H}_{16} \mathrm{~N}_{4} \mathrm{O}_{3}$ : 264.1221; found: 264.1222 . 
(S)-N-(3-Methyl-1-morpholino-1-oxobutan-2-yl)pyrazine-2-carboxamide (POA-L-Val-Mor, 5c)

Yield: 76\%; oil.

${ }^{1} \mathrm{H}$ NMR $\left(500 \mathrm{MHz}, \mathrm{CDCl}_{3}\right.$ ): $\delta=9.37(\mathrm{~s}, 1 \mathrm{H}), 8.76$ (br s, $\left.1 \mathrm{H}\right), 8.58$ (br s, $1 \mathrm{H}), 8.50(\mathrm{~d}, J=8.7 \mathrm{~Hz}, 1 \mathrm{H}), 5.00-4.97(\mathrm{~m}, 1 \mathrm{H}), 3.77-3.60(\mathrm{~m}$, $8 \mathrm{H}), 2.19-2.13(\mathrm{~m}, 1 \mathrm{H}), 1.05$ (d, J=6.6 Hz, $3 \mathrm{H}), 1.00$ (d, J=6.6 Hz, $3 \mathrm{H}$ ). ${ }^{13} \mathrm{C} \mathrm{NMR}\left(125 \mathrm{MHz}, \mathrm{CDCl}_{3}\right): \delta=169.8,163.0,147.3,144.3,142.7,66.7$, $66.6,53.2,46.3,42.4,31.7,19.7,17.5$.

HRMS (ESI): $m / z[M+\mathrm{H}]^{+}$calcd for $\mathrm{C}_{14} \mathrm{H}_{20} \mathrm{~N}_{4} \mathrm{O}_{3}$ : 292.1535; found: 292.1534

$N$-((2S,3S)-3-Methyl-1-morpholino-1-oxopentan-2-yl)pyrazine-2carboxamide (POA-L-Ile-Mor, 5d)

Yield: 70\%; oil.

${ }^{1} \mathrm{H}$ NMR $\left(500 \mathrm{MHz}, \mathrm{CDCl}_{3}\right): \delta=9.37(\mathrm{~s}, 1 \mathrm{H}), 8.76$ (br s, $\left.1 \mathrm{H}\right), 8.57$ (br s, $1 \mathrm{H}), 8.46(\mathrm{~d}, J=7.9 \mathrm{~Hz}, 1 \mathrm{H}), 5.02(\mathrm{t}, J=7.9 \mathrm{H}, 1 \mathrm{H}), 3.76-3.52(\mathrm{~m}, 8 \mathrm{H})$, 1.94-1.93 (m, $1 \mathrm{H}), 1.64-1.59(\mathrm{~m}, 1 \mathrm{H}), 1.23-1.16(\mathrm{~m}, 1 \mathrm{H}), 1.02-0.91$ $(\mathrm{m}, 6 \mathrm{H})$.

${ }^{13} \mathrm{C}$ NMR $\left(125 \mathrm{MHz}, \mathrm{CDCl}_{3}\right)$ : $\delta=170.3,163.0,147.5,144.5,142.9,67.0$, $66.9,52.8,46.7,42.7,38.4,24.4,16.1,11.4$.

HRMS (ESI): $m / z[\mathrm{M}+\mathrm{H}]^{+}$calcd for $\mathrm{C}_{15} \mathrm{H}_{22} \mathrm{~N}_{4} \mathrm{O}_{3}$ : 306.1694; found: 306.1692 .

(S)-N-(1-Morpholino-1-oxo-3-phenylpropan-2-yl)pyrazine-2-carboxamide (POA-L-Phe-Mor, 5e)

Yield: 78\%; gum.

${ }^{1} \mathrm{H}$ NMR $\left(500 \mathrm{MHz}, \mathrm{CDCl}_{3}\right): \delta=9.36(\mathrm{~s}, 1 \mathrm{H}), 8.75$ (br s, $1 \mathrm{H}$ ), 8.59-856 ( $\mathrm{m}, 2 \mathrm{H}), 7.33-7.26(\mathrm{~m}, 5 \mathrm{H}), 5.35-5.30(\mathrm{~m}, 1 \mathrm{H}), 3.61-3.32(\mathrm{~m}, 6 \mathrm{H})$, 3.20-3.09 (m, $2 \mathrm{H}), 3.00-2.89(\mathrm{~m}, 2 \mathrm{H})$.

${ }^{13} \mathrm{C}$ NMR $\left(125 \mathrm{MHz}, \mathrm{CDCl}_{3}\right): \delta=169.7,162.6,147.6,144.5,143.0$, 136.2, 129.8, 128.9, 127.6, 66.7, 66.2, 49.8, 46.3, 42.5, 40.4.

HRMS (ESI): $m / z[\mathrm{M}+\mathrm{H}]^{+}$calcd for $\mathrm{C}_{18} \mathrm{H}_{20} \mathrm{~N}_{4} \mathrm{O}_{3}$ : 340.1536; found: 340.1535 .

$\mathrm{N}$-(2-0xo-2-(piperidin-1-yl)ethyl)pyrazine-2-carboxamide (POAGly-Pip, 5f)

Yield: 74\%; colorless microcrystals; mp $123-125^{\circ} \mathrm{C}$.

${ }^{1} \mathrm{H}$ NMR $\left(500 \mathrm{MHz}, \mathrm{CDCl}_{3}\right): \delta=9.38(\mathrm{~s}, 1 \mathrm{H}), 8.77-8.74(\mathrm{~m}, 2 \mathrm{H}), 8.58$ (br s, $1 \mathrm{H}), 4.27(\mathrm{~d}, J=4.3 \mathrm{~Hz}, 2 \mathrm{H}), 3.63(\mathrm{t}, J=5.2 \mathrm{~Hz}, 2 \mathrm{H}), 3.41(\mathrm{t}$, $J=5.2 \mathrm{~Hz}, 2 \mathrm{H}), 1.69-1.59(\mathrm{~m}, 6 \mathrm{H})$.

${ }^{13} \mathrm{C}$ NMR $\left(125 \mathrm{MHz}, \mathrm{CDCl}_{3}\right): \delta=165.8,163.3,147.5,144.4,143.1,45.8$, 43.4, 41.4, 41.4, 26.4, 25.6, 24.6.

HRMS (ESI): $m / z[M+H]^{+}$calcd for $\mathrm{C}_{12} \mathrm{H}_{16} \mathrm{~N}_{4} \mathrm{O}_{2}: 248.1273$; found: 248.1275 .

(S)-N-(1-0xo-1-(piperidin-1-yl)propan-2-yl)pyrazine-2-carboxamide (POA-L-Ala-Pip, 5g)

Yield: 75\%; oil.

${ }^{1} \mathrm{H}$ NMR $\left(500 \mathrm{MHz}, \mathrm{CDCl}_{3}\right): \delta=9.28(\mathrm{~s}, 1 \mathrm{H}), 8.74(\mathrm{~d}, J=7.2 \mathrm{~Hz}, 1 \mathrm{H})$, 8.66 (br s, $1 \mathrm{H}), 8.50$ (br s, $1 \mathrm{H}), 5.04-4.98(\mathrm{~m}, 1 \mathrm{H}), 3.61-3.38(\mathrm{~m}$, $4 \mathrm{H}), 1.61-1.45(\mathrm{~m}, 6 \mathrm{H}), 1.37(\mathrm{~d}, J=6.9 \mathrm{~Hz}, 3 \mathrm{H})$.

${ }^{13} \mathrm{C}$ NMR $\left(125 \mathrm{MHz}, \mathrm{CDCl}_{3}\right): \delta=169.9,162.1,147.2,144.1,142.8,46.5$, $45.3,43.3,26.4,25.5,24.4,19.0$.

HRMS (ESI): $m / z[M+H]^{+}$calcd for $\mathrm{C}_{13} \mathrm{H}_{18} \mathrm{~N}_{4} \mathrm{O}_{2}$ : 262.1429; found: 262.1431 .
(S)-N-(3-Methyl-1-oxo-1-(piperidin-1-yl)butan-2-yl)pyrazine-2carboxamide (POA-L-Val-Pip, 5h)

Yield: $81 \%$; oil.

${ }^{1} \mathrm{H}$ NMR $\left(500 \mathrm{MHz}, \mathrm{CDCl}_{3}\right): \delta=9.31(\mathrm{~s}, 1 \mathrm{H}), 8.68$ (br s, $1 \mathrm{H}$ ), 8.52-8.49 (m, $2 \mathrm{H}), 5.00-4.97(\mathrm{~m}, 1 \mathrm{H}), 3.59-3.51(\mathrm{~m}, 4 \mathrm{H}), 2.12-2.05(\mathrm{~m}, 1 \mathrm{H})$, $1.61-150(\mathrm{~m}, 6 \mathrm{H}), 0.98$ (d, J=6.6 Hz, $3 \mathrm{H}), 0.91(\mathrm{~d}, J=6.6 \mathrm{~Hz}, 3 \mathrm{H})$.

${ }^{13} \mathrm{C} \mathrm{NMR}\left(125 \mathrm{MHz}, \mathrm{CDCl}_{3}\right): \delta=169.5,163.0,147.3,144.5,142.9,53.5$, 47.1, 43.4, 32.1, 26.7, 25.7, 24.6, 20.0, 17.5.

HRMS (ESI): $m / z[M+H]^{+}$calcd for $\mathrm{C}_{15} \mathrm{H}_{22} \mathrm{~N}_{4} \mathrm{O}_{2}$ : 290.1742; found: 290.1749 .

$N$-((2S,3S)-3-Methyl-1-oxo-1-(piperidin-1-yl)pentan-2-yl)pyrazine-2-carboxamide (POA-L-Ile-Pip, 5i)

Yield: 64\%; gum.

${ }^{1} \mathrm{H} \mathrm{NMR}\left(500 \mathrm{MHz}, \mathrm{CDCl}_{3}\right): \delta=9.32(\mathrm{~s}, 1 \mathrm{H}), 8.73(\mathrm{br} \mathrm{s}, 1 \mathrm{H}), 8.52(\mathrm{br} \mathrm{s}$, $1 \mathrm{H}), 8.43(\mathrm{~d}, J=7.9 \mathrm{~Hz}, 1 \mathrm{H}), 5.04(\mathrm{t}, J=7.9 \mathrm{H}, 1 \mathrm{H}), 3.54-3.49(\mathrm{~m}$, $4 \mathrm{H}), 1.87-1.85(\mathrm{~m}, 1 \mathrm{H}), 1.15-1.08(\mathrm{~m}, 8 \mathrm{H}), 0.92(\mathrm{~d}, J=7.3 \mathrm{~Hz}, 1 \mathrm{H})$, $0.79(\mathrm{~d}, J=7.3 \mathrm{~Hz}, 1 \mathrm{H})$.

${ }^{13} \mathrm{C}$ NMR $\left(125 \mathrm{MHz}, \mathrm{CDCl}_{3}\right): \delta=170.6,163.3,147.5,144.7,142.6,53.2$, 47.3, 43.6, 38.4, 26.5, 25.6, 24.3, 24.0, 16.0, 11.4.

HRMS (ESI): $m / z[M+H]^{+}$calcd for $\mathrm{C}_{16} \mathrm{H}_{24} \mathrm{~N}_{4} \mathrm{O}_{2}$ : 304.1896; found: 304.1899.

(S)-N-(1-0xo-3-phenyl-1-(piperidin-1-yl)propan-2-yl)pyrazine-2carboxamide (POA-L-Phe-Pip, 5j)

Yield: 79\%; oil.

${ }^{1} \mathrm{H} \mathrm{NMR}\left(500 \mathrm{MHz}, \mathrm{CDCl}_{3}\right): \delta=9.35$ (s, $1 \mathrm{H}$ ), 8.73 (br s, $1 \mathrm{H}$ ), 8.62-8.56 (m, $2 \mathrm{H}), 7.34-7.24(\mathrm{~m}, 5 \mathrm{H}), 5.40-5.35(\mathrm{~m}, 1 \mathrm{H}), 3.57-3.47(\mathrm{~m}, 4 \mathrm{H})$, 2.94-2.78 (m, $2 \mathrm{H}), 1.54-1.43(\mathrm{~m}, 6 \mathrm{H})$.

${ }^{13} \mathrm{C}$ NMR $\left(125 \mathrm{MHz}, \mathrm{CDCl}_{3}\right): \delta=169.1,162.4,147.4,144.4,142.9$, 138.0, 129.5, 128.6, 127.2, 52.5, 49.9, 46.3, 43.2, 26.2, 25.6, 24.4.

HRMS (ESI): $m / z[\mathrm{M}+\mathrm{H}]^{+}$calcd for $\mathrm{C}_{19} \mathrm{H}_{22} \mathrm{~N}_{4} \mathrm{O}_{2}$ : 338.1743; found: 338.1746 .

$\mathrm{N}$-(2-(4-Methylpiperazin-1-yl)-2-oxoethyl)pyrazine-2-carboxamide (POA-Gly-NMP, 5k)

Yield: 69\%; gum.

${ }^{1} \mathrm{H} \mathrm{NMR}\left(500 \mathrm{MHz}, \mathrm{CDCl}_{3}\right.$ ): $\delta=9.34$ (s, $\left.1 \mathrm{H}\right), 8.71$ (br s, $\left.1 \mathrm{H}\right), 8.68$ (br s, $1 \mathrm{H}), 8.54$ (br s, $1 \mathrm{H}), 4.26$ (d, J=4.3 Hz, $2 \mathrm{H}), 3.75-3.60$ (m, $4 \mathrm{H}), 2.42-$ $2.35(\mathrm{~m}, 4 \mathrm{H}), 2.31(\mathrm{~s}, 3 \mathrm{H})$.

${ }^{13} \mathrm{C}$ NMR $\left(125 \mathrm{MHz}, \mathrm{CDCl}_{3}\right): \delta=169.4,163.3,147.8,144.5,143.4,55.3$, 54.8, 46.4, 46.1, 45.9, 40.8.

HRMS (ESI): $m / z[M+H]^{+}$calcd for $\mathrm{C}_{12} \mathrm{H}_{17} \mathrm{~N}_{5} \mathrm{O}_{2}$ : 263.1385; found: 263.1386 .

(S)-N-(1-(4-Methylpiperazin-1-yl)-1-oxopropan-2-yl)pyrazine-2carboxamide (POA-L-Ala-NMP, 51)

Yield: 73\%; oil.

${ }^{1} \mathrm{H}$ NMR (500 MHz, $\mathrm{CDCl}_{3}$ ): $\delta=9.32(\mathrm{~s}, 1 \mathrm{H}), 8.72-8.70(\mathrm{~m}, 2 \mathrm{H}), 8.53$ (br s, $1 \mathrm{H}), 5.07-5.04(\mathrm{~m}, 1 \mathrm{H}), 3.72-3.51(\mathrm{~m}, 4 \mathrm{H}), 2.44-2.34(\mathrm{~m}, 4 \mathrm{H})$, $2.27(\mathrm{~s}, 3 \mathrm{H}), 1.42(\mathrm{~d}, \mathrm{~J}=6.7 \mathrm{~Hz}, 3 \mathrm{H})$.

${ }^{13} \mathrm{C}$ NMR $\left(125 \mathrm{MHz}, \mathrm{CDCl}_{3}\right): \delta=170.3,162.3,147.4,144.3,142.9,55.1$, 54.6, 46.0, 45.4, 45.3, 42.2, 19.1 .

HRMS (ESI): $m / z[M+H]^{+}$calcd for $\mathrm{C}_{13} \mathrm{H}_{19} \mathrm{~N}_{5} \mathrm{O}_{2}:$ 277.1536; found: 277.1541 . 
(S)-N-(3-Methyl-1-(4-methylpiperazin-1-yl)-1-oxobutan-2-yl)pyrazine-2-carboxamide (POA-L-Val-NMP, 5m)

Yield: 62\%; oil.

${ }^{1} \mathrm{H}$ NMR (500 MHz, $\mathrm{CDCl}_{3}$ ): $\delta=9.37(\mathrm{~s}, 1 \mathrm{H}), 8.75$ (br s, $1 \mathrm{H}$ ), 8.57 (br s, $1 \mathrm{H}), 8.52(\mathrm{~d}, J=8.9 \mathrm{~Hz}, 1 \mathrm{H}), 5.03-5.00(\mathrm{~m}, 1 \mathrm{H}), 3.76-3.61(\mathrm{~m}, 4 \mathrm{H})$, 2.46-2.38 (m, $4 \mathrm{H}), 2.31$ (s, $3 \mathrm{H}), 2.17-2.13(\mathrm{~m}, 1 \mathrm{H}), 1.04(\mathrm{~d}, J=6.7 \mathrm{~Hz}$, $3 \mathrm{H}), 0.98(\mathrm{~d}, J=6.7 \mathrm{~Hz}, 3 \mathrm{H})$

${ }^{13} \mathrm{C}$ NMR $\left(125 \mathrm{MHz}, \mathrm{CDCl}_{3}\right): \delta=169.8,163.3,147.5,144.4,143.0,55.4$, 54.9, 53.6, 46.2, 46.0, 42.3, 32.1, 20.1, 17.7 .

HRMS (ESI): $m / z[M+H]^{+}$calcd for $\mathrm{C}_{15} \mathrm{H}_{23} \mathrm{~N}_{5} \mathrm{O}_{2}$ : 305.1852; found: 305.1860 .

$N$-((2S,3S)-3-Methyl-1-(4-methylpiperazin-1-yl)-1-oxopentan-2yl)pyrazine-2-carboxamide (POA-L-Ile-NMP, 5n)

Yield: 64\%; oil.

${ }^{1} \mathrm{H} \mathrm{NMR}\left(500 \mathrm{MHz}, \mathrm{CDCl}_{3}\right): \delta=9.32(\mathrm{~s}, 1 \mathrm{H}), 8.70(\mathrm{br} \mathrm{s}, 1 \mathrm{H}), 8.52(\mathrm{br} \mathrm{s}$, $1 \mathrm{H}), 8.45$ (d, J=7.8 Hz, $1 \mathrm{H}), 5.01(\mathrm{t}, J=7.8 \mathrm{H}, 1 \mathrm{H}), 3.72-3.60(\mathrm{~m}, 4 \mathrm{H})$, 2.42-2.36 (m, $4 \mathrm{H}), 2.27(\mathrm{~s}, 3 \mathrm{H}), 1.89-1.88(\mathrm{~m}, 1 \mathrm{H}), 1.57-1.53(\mathrm{~m}$, $1 \mathrm{H}), 1.18-1.13(\mathrm{~m}, 1 \mathrm{H}), 0.98-0.86(\mathrm{~m}, 6 \mathrm{H})$.

${ }^{13} \mathrm{C} \mathrm{NMR}\left(125 \mathrm{MHz}, \mathrm{CDCl}_{3}\right): \delta=169.9,162.9,147.4,144.4,142.9,55.3$, $54.7,52.9,46.0,42.2,38.4,24.3,16.1,11.4$.

HRMS (ESI): $m / z[M+H]^{+}$calcd for $\mathrm{C}_{16} \mathrm{H}_{25} \mathrm{~N}_{5} \mathrm{O}_{2}: 319.2005$; found: 319.2000 .

(S)-N-(1-(4-Methylpiperazin-1-yl)-1-oxo-3-phenylpropan-2yl)pyrazine-2-carboxamide (POA-L-Phe-NMP, 5o)

Yield: 71\%; oil.

${ }^{1} \mathrm{H}$ NMR $\left(500 \mathrm{MHz}, \mathrm{CDCl}_{3}\right.$ ): $\delta=9.34(\mathrm{~s}, 1 \mathrm{H}), 8.73$ (br s, $1 \mathrm{H}$ ), 8.58-854 $(\mathrm{m}, 2 \mathrm{H}), 7.30-7.19(\mathrm{~m}, 5 \mathrm{H}), 5.30-5.24(\mathrm{~m}, 1 \mathrm{H}), 3.72-3.61(\mathrm{~m}, 4 \mathrm{H})$, 3.20-3.09 (m, $2 \mathrm{H}), 2.47-2.38$ (m, $4 \mathrm{H}), 2.24(\mathrm{~s}, 3 \mathrm{H}$ ).

${ }^{13} \mathrm{C}$ NMR $\left(125 \mathrm{MHz}, \mathrm{CDCl}_{3}\right): \delta=170.1,162.8,147.5,144.7,143.2$, $136.5,129.3,128.7,127.2,55.3,54.7,53.5,46.5,46.2,42.6,40.8$.

HRMS (ESI): $m / z[M+H]^{+}$calcd for $\mathrm{C}_{19} \mathrm{H}_{23} \mathrm{~N}_{5} \mathrm{O}_{2}$ : 353.1853; found: 353.1850.

N-(2-0xo-2-(pyrrolidin-1-yl)ethyl)pyrazine-2-carboxamide (POAGly-Pyr, 5p)

Yield: $81 \%$; colorless microcrystals; $\mathrm{mp} 148-150{ }^{\circ} \mathrm{C}$.

${ }^{1} \mathrm{H}$ NMR $\left(500 \mathrm{MHz}, \mathrm{CDCl}_{3}\right): \delta=9.31(\mathrm{~s}, 1 \mathrm{H}), 8.68-8.64(\mathrm{~m}, 2 \mathrm{H}), 8.52$ (br s, $1 \mathrm{H}), 4.15$ (d, J=4.3 Hz, $2 \mathrm{H}), 3.50-3.40$ (m, $4 \mathrm{H}), 2.00-1.78(\mathrm{~m}$, $4 \mathrm{H})$.

${ }^{13} \mathrm{C}$ NMR $\left(125 \mathrm{MHz}, \mathrm{CDCl}_{3}\right): \delta=166.1,163.3,147.4,144.3,143.0,68.1$, 46.2, 45.7, 42.1, 26.1, 24.3.

HRMS (ESI): $m / z[M+H]^{+}$calcd for $\mathrm{C}_{11} \mathrm{H}_{14} \mathrm{~N}_{4} \mathrm{O}_{2}: 234.1117$; found: 234.1121.

(S)-N-(1-0xo-1-(pyrrolidin-1-yl)propan-2-yl)pyrazine-2-carboxamide (POA-L-Ala-Pyr, 5q)

Yield: 79\%; oil.

${ }^{1} \mathrm{H} \mathrm{NMR} \mathrm{(500} \mathrm{MHz,} \mathrm{CDCl}_{3}$ ): $\delta=9.29(\mathrm{~s}, 1 \mathrm{H}), 8.67$ (br s, $1 \mathrm{H}$ ), 8.59 (br s, $1 \mathrm{H}), 8.49$ (br s, $1 \mathrm{H}), 4.89-4.85$ (m, $1 \mathrm{H}), 3.64-3.40$ (m, $4 \mathrm{H}), 1.97-$ $1.82(\mathrm{~m}, 4 \mathrm{H}), 1.41(\mathrm{~d}, J=6.4 \mathrm{~Hz}, 3 \mathrm{H})$.

${ }^{13} \mathrm{C} \mathrm{NMR}\left(125 \mathrm{MHz}, \mathrm{CDCl}_{3}\right): \delta=170.4,162.4,147.3,144.3,142.9,47.0$, 46.6, 46.2, 26.2, 24.2, 18.4.

HRMS (ESI): $m / z[M+H]^{+}$calcd for $\mathrm{C}_{12} \mathrm{H}_{16} \mathrm{~N}_{4} \mathrm{O}_{2}: 248.1271$; found: 248.1279 .
(S)-N-(3-Methyl-1-oxo-1-(pyrrolidin-1-yl)butan-2-yl)pyrazine-2carboxamide (POA-L-Val-Pyr, 5r)

Yield: 82\%; oil.

${ }^{1} \mathrm{H} \mathrm{NMR}\left(500 \mathrm{MHz}, \mathrm{CDCl}_{3}\right): \delta=9.32(\mathrm{~s}, 1 \mathrm{H}), 8.69$ (br s, $1 \mathrm{H}$ ), 8.52 (br s, $1 \mathrm{H}), 8.38(\mathrm{~d}, J=8.2 \mathrm{~Hz}, 1 \mathrm{H}), 4.74(\mathrm{t}, J=8.2 \mathrm{~Hz}, 1 \mathrm{H}), 3.77-3.39(\mathrm{~m}, 4 \mathrm{H})$, 2.18-2.11 (m, $1 \mathrm{H}), 1.98-1.82(\mathrm{~m}, 4 \mathrm{H}), 1.00$ (d, J=6.6 Hz, $3 \mathrm{H}), 0.97$ (d, $J=6.6 \mathrm{~Hz}, 3 \mathrm{H})$

${ }^{13} \mathrm{C}$ NMR $\left(125 \mathrm{MHz}, \mathrm{CDCl}_{3}\right): \delta=169.9,163.0,147.4,144.5,142.9,56.1$, 47.0, 46.1, 31.8, 26.2, 24.4, 19.8, 18.1 .

HRMS (ESI): $m / z[M+H]^{+}$calcd for $\mathrm{C}_{14} \mathrm{H}_{20} \mathrm{~N}_{4} \mathrm{O}_{2}$ : 276.1586; found: 276.1582 .

$N$-((2S,3S)-3-Methyl-1-oxo-1-(pyrrolidin-1-yl)pentan-2-yl)pyrazine-2-carboxamide (POA-L-Ile-Pyr, 5s)

Yield: 74\%; oil.

$\left.{ }^{1} \mathrm{H} \mathrm{NMR} \mathrm{(500} \mathrm{MHz,} \mathrm{CDCl}_{3}\right): \delta=9.34(\mathrm{~s}, 1 \mathrm{H}), 8.68$ (br s, $1 \mathrm{H}$ ), 8.57 (br s, $1 \mathrm{H}), 8.49(\mathrm{~d}, J=7.8 \mathrm{~Hz}, 1 \mathrm{H}), 5.04(\mathrm{t}, J=7.8 \mathrm{H}, 1 \mathrm{H}), 3.66-3.42(\mathrm{~m}, 4 \mathrm{H})$, 2.01-1.88 (m, $5 \mathrm{H}), 1.62-1.58$ ( $\mathrm{m}, 1 \mathrm{H}), 1.17-1.12$ (m, $1 \mathrm{H}), 1.01-0.89$ $(\mathrm{m}, 6 \mathrm{H})$

${ }^{13} \mathrm{C}$ NMR $\left(125 \mathrm{MHz}, \mathrm{CDCl}_{3}\right): \delta=170.3,163.1,147.6,144.2,142.5,52.9$, 45.7, 42.6, 38.1, 25.0, 24.8, 24.5, 16.2, 11.7 .

HRMS (ESI): $m / z[M+H]^{+}$calcd for $\mathrm{C}_{15} \mathrm{H}_{22} \mathrm{~N}_{4} \mathrm{O}_{2}$ : 290.1743; found: 290.1746 .

(S)-N-(1-0xo-3-phenyl-1-(pyrrolidin-1-yl)propan-2-yl)pyrazine-2carboxamide (POA-L-Phe-Pyr, 5t)

Yield: 73\%; oil.

${ }^{1} \mathrm{H}$ NMR $\left(500 \mathrm{MHz}, \mathrm{CDCl}_{3}\right): \delta=9.33(\mathrm{~s}, 1 \mathrm{H}), 8.73$ (br s, $1 \mathrm{H}$ ), 8.59-855 $(\mathrm{m}, 2 \mathrm{H}), 7.35-7.23(\mathrm{~m}, 5 \mathrm{H}), 5.14-5.09(\mathrm{~m}, 1 \mathrm{H}), 3.75-3.34(\mathrm{~m}, 4 \mathrm{H})$, 3.03-2.93 (m, $2 \mathrm{H}), 2.03-1.72(\mathrm{~m}, 4 \mathrm{H})$.

${ }^{13} \mathrm{C}$ NMR $\left(125 \mathrm{MHz}, \mathrm{CDCl}_{3}\right): \delta=169.0,162.3,147.3,144.2,142.8$, 136.2, 129.2, 128.3, 126.6, 52.5, 45.9, 45.8, 39.7, 24.0, 23.8.

HRMS (ESI): $m / z[M+H]^{+}$calcd for $\mathrm{C}_{18} \mathrm{H}_{20} \mathrm{~N}_{4} \mathrm{O}_{2}$ : 324.1588; found: 324.1585 .

\section{Acknowledgment}

We thank the Augusta University and Pamplin Student Research \& Travel Fund for financial support.

\section{Supporting Information}

Supporting information for this article is available online at https://doi.org/10.1055/s-0036-1590800.

\section{References}

(1) Mitchison, D. A. Tubercle 1985, 66, 219.

(2) Joshi, R. R.; Barchha, A.; Khedkar, V. M.; Pissurlenkar, R. R. S.; Sarkar, S.; Sarkar, D.; Joshi, R. R.; Joshi, R. A.; Shah, A. K.; Coutinho, E. C. Chem. Biol. Drug Des. 2015, 85, 201.

(3) Rivers, E. C. R.; Mancera, L. Drug Discovery Today 2008, 13, 1090.

(4) World Health Organization. Global Tuberculosis, Report 2013. WHO/HTM/TB/ 2013, 11. 
(5) Goletti, D.; Weissman, D.; Jackson, R. W.; Graham, N. M.; Vlahov, D.; Klein, R. S.; Munsiff, S. S.; L'Ortona, L.; Cauda, R.; Fauci, A. S. J. Immunol. 1996, 157, 1271.

(6) Ma, Z.; Ginsberg, A. M.; Spigelman, M. Antimycobacterium agents, In Comprehensive Medicinal Chemistry II; Taylor, J. B.; Triggle, D. J., Eds.; Elsevier: Oxford, 2006, Vol. 7, 699.

(7) Kremer, L.; Besra, G. S. Expert Opin. Invest. Drugs 2002, 11, 1033.

(8) World Health Organization (WHO), WHO Report 2010: Global Tuberculosis Control, 2010.

(9) Zhang, Y.; Mitchison, D. Int. J. Tuberc. Lung Dis. 2003, 7, 6.

(10) Van den Boogaard, J.; Kibiki, G. S.; Kisanga, E. R.; Boeree, M. J.; Aarnoutse, R. E. Antimicrob. Agents Chemother. 2009, 53, 849.

(11) Reddy, V. M.; Nadadhur, G.; Daneluzzi, D.; Dimova, V.; Gangadharam, P. R. Antimicrob. Agents Chemother. 1995, 39, 2320.

(12) Chitre, T. S.; Asgaonkar, K. D.; Miniyar, P. B.; Dharme, A. B.; Arkile, M. A.; Yeware, A.; Sarkar, D.; Khedkar, V. M.; Jha, P. C. Bioorg. Med. Chem. Lett. 2016, 26, 2224.

(13) Jardosh, H. H.; Patel, M. P. Eur. J. Med. Chem. 2013, 65, 348.
(14) Saha, R.; Alam, M. M.; Akhter, M. RSC Adv. 2015, 5, 12807.

(15) Simoes, M. F.; Valente, E.; Gomez, M. J. R.; Anes, E.; Constantino, L. Eur. J. Pharm. Sci. 2009, 37, 257.

(16) Mohamed, T.; Zhao, X.; Habib, L. K.; Yang, J.; Rao, P. P. N. Bioorg. Med. Chem. 2011, 19, 2269.

(17) Panda, S. S.; Bajaj, K.; Meyers, M. J.; Sverdrup, F. M.; Katritzky, A. R. Org. Biomol. Chem. 2012, 10, 8985.

(18) Ibrahim, M. A.; Panda, S. S.; Birs, A. S.; Serrano, J. C.; Gonzalez, C. F.; Alamry, K. A.; Katritzky, A. R. Bioorg. Med. Chem. Lett. 2014, 24, 1856.

(19) Panda, S. S.; Naumov, R. N.; Asiri, A. M.; Katritzky, A. R. Synthesis 2014, 46, 1511.

(20) Panda, S. S.; Liaqat, S.; Girgis, A. S.; Samir, A.; Hall, C. D.; Katritzky, A. R. Bioorg. Med. Chem. Lett. 2015, 25, 3816.

(21) Panda, S. S.; Detistov, O. S.; Girgis, A. S.; Mohapatra, P. P.; Samir, A.; Katritzky, A. R. Bioorg. Med. Chem. Lett. 2016, 26, 2198.

(22) Panda, S. S.; Hall, C. D.; Scriven, E.; Katritzky, A. R. Aldrichimica Acta 2013, 46, 43. 